\title{
Personalized Pain Medicine: The Clinical Value of Psychophysical Assessment of Pain Modulation Profile
}

\author{
Yelena Granovsky, Ph.D.* and David Yarnitsky, M.D. \\ Department of Neurology, Rambam Medical Center, and Clinical Neurophysiology Lab, Faculty of \\ Medicine, Technion - Israel Institute of Technology, Haifa, Israel
}

\begin{abstract}
Experimental pain stimuli can be used to simulate patients' pain experience. We review recent developments in psychophysical pain testing, focusing on the application of the dynamic tests-conditioned pain modulation (CPM) and temporal summation (TS). Typically, patients with clinical pain of various types express either less efficient CPM or enhanced TS, or both. These tests can be used in prediction of incidence of acquiring pain and of its intensity, as well as in assisting the correct choice of analgesic agents for individual patients. This can help to shorten the commonly occurring long and frustrating process of adjusting analgesic agents to the individual patients. We propose that evaluating pain modulation can serve as a step forward in individualizing pain medicine.
\end{abstract}

KEY WORDS: Conditioned pain modulation, individualized medicine, pain, psychophysics, temporal summation

\section{INTRODUCTION}

Since pain is a subjective and complex experience, researchers have found substantial difficulties in measuring it and, consequently, in promoting research into it. One of the common approaches to bypass this difficulty is the use of experimental pain stimuli, given in well-defined and quantitative ways.

\footnotetext{
Abbreviations: CPM, conditioned pain modulation; DNIC, diffused noxious inhibitory control; NMDA, N-methyl-Daspartate; NPS, numerical pain score; QST, quantitative sensory testing; SNRIs, serotonin-noradrenaline re-uptake inhibitors; TS, temporal summation; VAS, visual analog scale.

Citation: Granovsky Y, Yarnitsky D. Personalized Pain Medicine: The Clinical Value of Psychophysical Assessment of Pain Modulation Profile. Rambam Maimonides Med J 2013;4 (4):e0024. doi:10.5041/RMMJ.10131

Copyright: (C) 2013 Granovsky and Yarnitsky. This is an open-access article. All its content, except where otherwise noted, is distributed under the terms of the Creative Commons Attribution License

(http://creativecommons.org/licenses/by/3.o), which permits unrestricted use, distribution, and reproduction in any medium, provided the original work is properly cited.
}

Conflict of interest: No potential conflict of interest relevant to this article was reported.

* To whom correspondence should be addressed. E-mail: y_granovsky@rambam.health.gov.il 
The measures of pain thresholds and pain tolerance were the main parameters sought over many years. Experience has taught us that thresholds are useful parameters for assessment of sensory deficit, as part of diagnosing nerve damage. This way, elevated thresholds for perceiving the sensations of warm, cold, and mechanical and electrical stimuli are often used in assessing severity of neural damage, such that a high threshold indicates more severe neuropathic damage. This is especially important for damage to small fibers, whose function is not depicted by standard nerve conduction-electromyography tests. Thresholds for painful stimuli, similarly, can identify loss of function, but, since part of the pain inventory of symptoms includes gain of function, such as allodynia and hyperalgesia, these thresholds can be increased or decreased, lowering their sensitivity in identifying the nerve damage. Further, thresholds are not necessarily correlated with the pain experience patients undergo. The best example would be the painful diabetic neuropathy, where the patients demonstrate a combination of peripheral sensory loss and hyperalgesia at the initial stage of disease; in contrast, at the advanced stage the patients exhibit both sensory loss and hypoalgesia, as can be assessed via quantitative sensory testing (QST).

Magnitude estimation of painful stimuli given at supra-threshold intensity is a different approach to the use of experimental stimuli in the pain lab. Practically, a painful stimulus is administered, whose intensity is higher than the pain threshold for that individual, and lower than the pain tolerance. A rating on a visual analog scale (VAS) or a numerical pain score (NPS) is given by the patient. Several studies have shown significant association between supra-threshold pain obtained from patients before surgery, and the levels of their acute post-operative pain. ${ }^{1-6}$ More specifically, the association of presurgery perception of the experimental pain stimuli and the post-operative pain intensity was established for thermal, mechanical, and electrical sensory modalities in gynecology, back, and knee surgeries, as well as in thoracotomy, cholecystectomy, and herniotomy, including laparoscopy surgeries. However, the above-mentioned parameters of pain threshold, supra-threshold pain estimation, and pain tolerance are usually related to as the static parameters of experimental pain, which isolate a single point of the pain experience of the patient.
A further step forward in pain psychophysics is the use of the dynamic stimulation protocols that give an array of stimuli, in varying combinations, to evoke a process of pain modulation. Pain inhibition is measured by the diffused noxious inhibitory control (DNIC) effect. This is a physiological phenomenon described in the late 1970s in animals, expressing the fact that painful stimuli exert inhibitory effects over other painful stimuli.7,8 Thus, if we take it to the human pain assessment, if a subject is asked to rate the intensity of a certain test stimulus and then given the combination of a conditioning pain and a repeated same test stimulus, the perceived intensity of the second test stimulus will generally be lower than when given alone. The term conditioned pain modulation (CPM) has recently been coined for the psychophysical protocols 9 that explore the DNIC phenomenon (Figure 1) and reflects the function of the descending tracts that control and modulate pain perception. These tracts, whose activity is initiated in the brainstem pain-controlling centers, are influenced by cerebral (the top-down effect) as well as up-going painful stimuli (bottom-up) and can exert either inhibition or facilitation on the spinal second-order neurons. Descending pain inhibition that underlies the CPM response is based on a spino-bulbar-spinal loop $7,8,10,11$ that involves serotonergic and noradrenergic neurotransmission. ${ }^{12-14}$ The aforementioned neurotransmission construct of the CPM response suggests augmentation of the descending inhibition leading to anti-nociception by increase of synaptic levels of noradrenaline and serotonin..$^{15}$

Pain facilitation is measured using the temporal summation (TS) protocol, where a series of identical stimuli is given and NPS obtained along the series. The common response is an increase in pain ratings along the series, representing the physiological phenomenon of wind-up-the sensitization of nociceptors in response to intense activation. TS represents neurophysiologic processes induced by excessive activation of $\mathrm{N}$-methyl-D-aspartate (NMDA) receptors of the second-order neurons, in response to intensive nociceptive input, and its expression depends on flow of $\mathrm{Ca}^{2+}$ ions into the neuronal cytoplasm. ${ }^{16}$ Thus, neuronal wind-up subsequent to the enhanced $\mathrm{Ca}^{2+}$ influx-dependent release of glutamate, norepinephrine, and substance $\mathrm{P}$ may serve as a target for the agents that are expected to diminish this central neuronal hyperexcitability. In other words, agents that target the 


\section{CPM}

\section{Conditioned Pain Modulation, the DNIC-like Phenomenon}

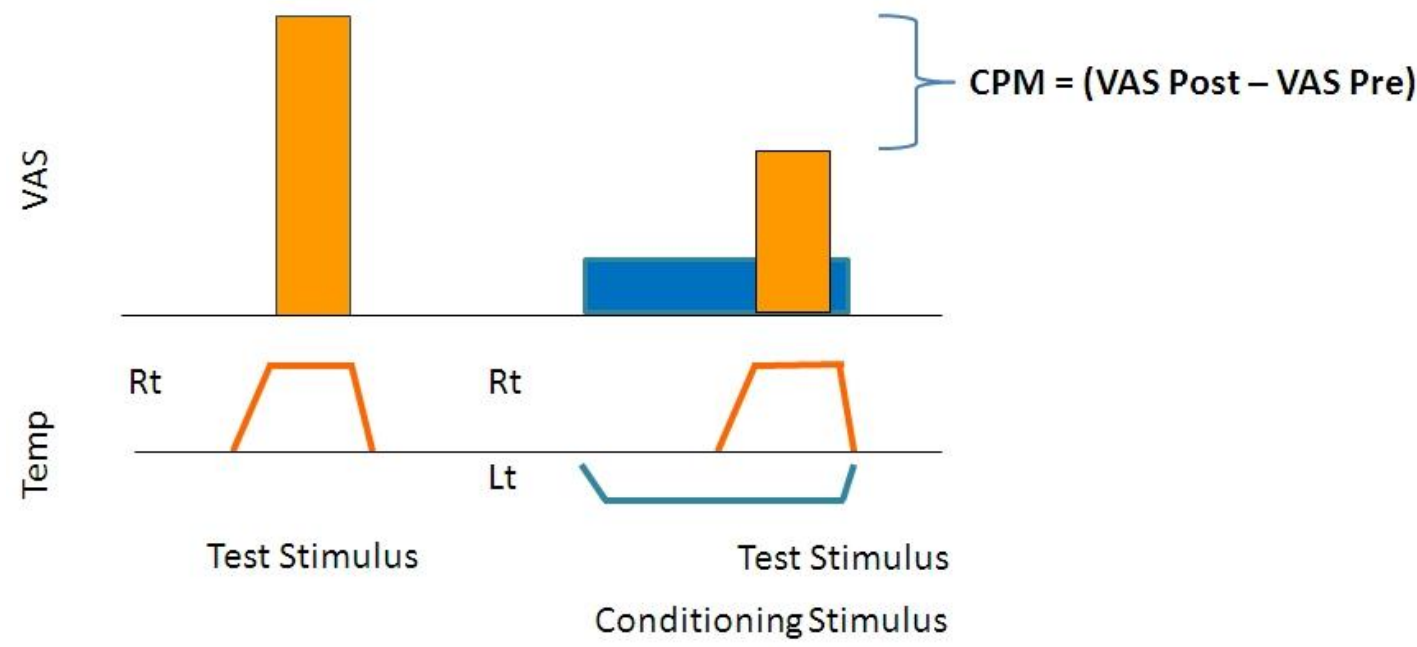

Figure 1. An Example of a Conditioned Pain Modulation (CPM) Test Protocol.

$\mathrm{Ca}^{2+}$ influx may reduce enhanced TS and alleviate pain.

These dynamic tests induce a process of modulation and are believed to reflect the "real-life" modulation exerted by patients when exposed to clinical pain. There is a large body of data showing differences between pain modulation states in patients suffering from idiopathic and other pain syndromes as compared to the healthy controls:

- Fibromyalgia. Various pain modalities applied for the noxious conditioning stimulation, by ischemic, contact heat, or cold noxious water, were non-efficient in increasing pain thresholds or reducing experimental supra-threshold pain magnitudes. ${ }^{17-19}$ Evidence for abnormal TS includes enhanced pain summation in response to repeated heat taps and repeated muscle taps delivered at a remote body area, as well as prolonged and enhanced painful after-sensations. Moreover, magnitudes of enhanced aftersensations were predictive of patients' ongoing clinical pain. ${ }^{20-22}$

- Irritable bowel syndrome. The experimentally induced visceral or cold water pain was not effective in reducing ongoing rectal pain or the perception of noxious heat. ${ }^{23-25}$

- Headache. Facilitation, rather than normally occurring inhibition, of nociceptive reflex was observed in migraine patients conditioned by noxious cold water. ${ }^{26}$ In line with this, in chronic tension-type headache patients, conditioning by tonic muscle pain failed to reduce the responses to electrical pain as recorded by somatosensory event-related potentials over the scalp. ${ }^{27}$ In the psychophysical domain, these patients demonstrated less efficient CPM in terms of lower increase in the electrical pain threshold during the exposure to conditioning pain, ${ }^{28}$ as well as significant waning of the CPM at the repeated application. ${ }^{29}$ Increased TS was found in migraine patients for repeated mechanical and electrical noxious stimuli delivered at the periorbital area as well as at a remote body site. Moreover, enhanced TS was demonstrated in association with more severe clinical parameters of disease and tended to normalize with time elapsed since last migraine attack. ${ }^{30}$

- Temporo-mandibular disorder. Submaximal effort tourniquet application as the conditioning stimulus was found non-efficient in reducing the clinical pain in these patients. ${ }^{11}$ These patients also responded with increased TS to repeated heat and to repeated mechanical noxious stimuli delivered on local and on remote from the painful body sites. ${ }^{22-35}$

- Osteoarthritis. Patients with knee and with hip osteoarthritis demonstrated less efficient CPM as assessed by the effect of experimental or ongoing clinical pain on pressure pain thresholds. ${ }^{36-40} \mathrm{In}$ addition, they demonstrated significant enhancement of TS to noxious pressure as well as to 
noxious heat stimuli at the site of inflammation and at remote body regions. ${ }^{41}$

- Whiplash. Results of a recent study raised evidence for impaired descending pain inhibition in chronic whiplash patients such that the application of ischemic pain as conditioning stimulus did not diminish the perception of pressure pain stimuli. ${ }^{2}$ In line with deficient endogenous pain inhibition, widespread deep tissue hyperalgesia in chronic whiplash was associated with enhanced TS to pressure pain stimuli.43,44

Consequently, the term "pro-nociceptive" is commonly used to describe, at the clinical level, the pain modulation profile of patients suffering from the idiopathic pain disorders. As can be seen from the aforementioned literature overview, these patients can express less efficient CPM, enhanced TS, or both, at psychophysical and neurophysiological levels, as compared to healthy subjects (Figure 2). The exact interrelations between inhibitory and facilitatory pain modulation systems in the clinical arena are still unclear. The reverse situation, an "anti-nociceptive" profile, is less known to us; most likely it represents an inherent or medication-induced resistance to pain. Likely examples would be the pain reduction in migraine patients in response to preventive treatment, and prevention of post-surgical pain by pre-emptive analgesic treatment.

The above-mentioned cross-sectional studies do not disclose whether the interrelations between the modulation state and the presence of the various pain syndromes are causative, and, if so, which one is primary to the other; it could be, on one hand, that a pre-existing facilitatory modulation state leads to the establishment of the pro-nociceptive profile and the acquisition of the idiopathic pain syndromes, or, on the other, that presence of the pain syndrome caused a change in modulation state and profile, shifting it toward the pro-nociception.

In an attempt to discern these potential causative relations, we explored these relationships in a longitudinal study performed in our lab, where prethoracotomy, pain-free patients were examined with the battery of psychophysical tests, including assessment of their pain modulation. The patients were followed up 1 year for acquisition of pain after surgery. 45 The results of this study confirmed our hypothesis that the baseline, pre-surgery CPM efficiency correlated with the intensity of postoperative pain. Moreover, among various demographic and psychophysics parameters (pain thresholds and supra-threshold pain), CPM efficiency was found to be the sole predictor of chronic postthoracotomy pain such that less efficient CPM patients had higher risk of development of chronic post-surgery pain and higher pain intensity. This reasonably establishes causative relations, at least in one direction, with pain modulation as a pathogenetic factor for future clinical pain. Results were later reproduced by Landau et al. and Wilder-Smith et al. for cesarean section and major abdominal surgery patients, respectively.46,47 Another interesting piece of evidence supporting "deficient pain inhibition = more pain acquisition" causative relations came from a recent animal-based study that shows the efficient engagement of descending inhibition to be a protection against the development of chronic neuropathic pain. 48

\section{The Nociception Spectrum}

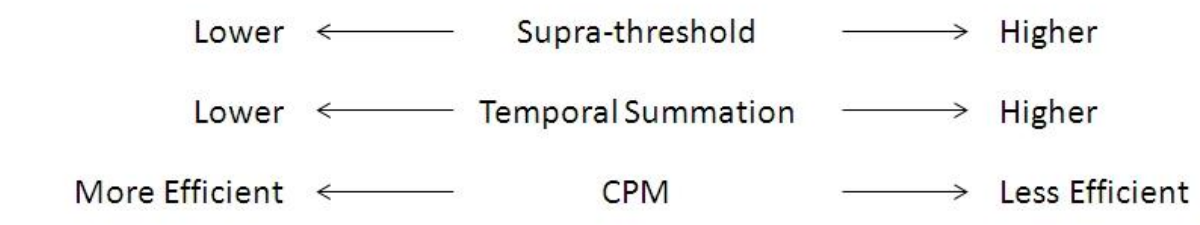

Anti-nociceptive

Pro-nociceptive

Figure 2. The Expression of Psychophysical Tests along the Pain Modulation Profile. 
A further advancement in the lab-to-clinic perusal of pain modulation is in the treatment of pain. Since pain modulation plays a role in pain acquisition, it should affect the relief of pain as well. Our assumption was that pain should be treated by "fixing" the dysfunctional pain modulation parameter of the individual patient. This way, patients with less efficient CPM should benefit more from serotonin-noradrenaline re-uptake inhibitors (SNRIs), which augment descending inhibition by spinal monoamine re-uptake inhibition, than patients whose CPM is already efficient. Similarly, those patients with enhanced TS should benefit more from gabapentinoids, inhibiting central neuronal sensitization, than those with nonenhanced pain summation. We examined CPM and TS in 30 painful diabetic neuropathy patients and found that among other psychophysical factors CPM predicted the efficacy of duloxetine, an SNRI; patients with less efficient pre-treatment CPM expressed high treatment efficacy in terms of pain reduction, while those with efficient CPM did not gain from the drug. 49 Further, for the former group, an improvement in CPM was found along with pain reduction, while no change in CPM was found for the latter group. Importantly, the CPM remained the only significant predictor for the duloxetine-induced pain relief after controlling for initial clinical pain, pre-treatment level of depression, neuropathy severity, and the placebo effect. On a similar note, Lavand'homme et al. published an abstract in 2009 on the use of ketamine in post-cesarean pain-they found that those patients with enhanced TS to repeated mechanical stimuli, tested pre-operatively, gained more analgesia from ketamine, an NMDA receptor blocker expected to reduce central neuronal sensitization, while those with non-enhanced pain summation did not benefit from the drug. $5^{\circ}$ It thus seems that the dysfunctional modulation state can be instrumental in the choice of drug for pain alleviation. This is a step forward toward individualized pain medicine.

A further question pertaining to pain modulation is whether it is flexible, or unchanged throughout life. A study on osteoarthritis patients undergoing hip replacement surgery showed an improvement in CPM, along with pain alleviation. ${ }^{36}$ It is noted that this was obtained for only one of several CPM protocols used in that study, a finding that highlights the need for additional studies on the interrelations between various testing protocols of pain modulation that yield varying results. Similar results were reported for patients undergoing knee replacement surgery. 39 These post-surgical results, together with our post-medication results reported above on diabetic neuropathy, suggest that pain modulation is a dynamic feature that probably tends to become pro-nociceptive during pain and to shift back upon alleviation of the pain.

Obviously, a pain modulation profile depends on many factors: 1) genetic factors, 2) environmentally influenced psychosocial factors, 3 ) the specifications of the pathology generating clinical pain, and 4) the pharmacological agents used to prevent or treat pain. Studies in recent years are trying to integrate psychophysical as well as genetic, neurophysiological, imaging, and other factors in exploring the pain phenomenon. A few recent examples follow: healthy subjects with low expression of serotonin transporter gene demonstrated less efficient CPM effect on pressure pain threshold and noxious heat.51,52 In the neurophysiology domain, a painevoked potentials-based source localization study showed reduced prefrontal cortical activity that was associated with altered pain inhibitory modulation in migraine patients. 53 A recent neuroimaging study characterized the CPM response as associated with reduced hemodynamic responses in classical painresponsive areas; furthermore, the CPM efficiency was associated with strength of functional connectivity between various structures on brain endogenous analgesia system. 54 Finally, there is an important integrative study by Loggia et al. who showed a "triple interaction" between the pain psychophysics, the activation in pain modulatory structures as measured by functional magnetic resonance imaging technique, and the genetics of catecholamine turnover. 55

Needless to say, further work is required in improving the protocols used for the dynamic psychophysics tests for individual pain assessment, to optimize their reliability, sensitivity, and specificity in describing the clinical pain events, finding the specific test paradigms for specific clinical questions or pathologies, and characterizing the relationships between the various pain testing paradigms. Use of new modalities of exploring the individual pain modulation capabilities, or new neuromodulatory technologies such as repetitive transcranial magnetic stimulation or novel transcranial direct-current stimulation, in combination with psychophysical test paradigms, is a promising new avenue for research in the pain field. 


\section{REFERENCES}

1. Bisgaard T, Klarskov B, Rosenberg J, Kehlet H. Characteristics and prediction of early pain after laparoscopic cholecystectomy. Pain 2001;90:261-9. Full Text

2. Granot M, Lowenstein L, Yarnitsky D, Tamir A, Zimmer EZ. Postcesarean section pain prediction by preoperative experimental pain assessment. Anesthesiology 2003;98:1422-6. Full Text

3. Pan PH, Coghill R, Houle TT, et al. Multifactorial preoperative predictors for postcesarean section pain and analgesic requirement. Anesthesiology 2006;104: 417-25. Full Text

4. Weissman-Fogel I, Granovsky Y, Crispel Y, et al. Enhanced presurgical pain temporal summation response predicts post-thoracotomy pain intensity during the acute postoperative phase. J Pain 2009; 10:628-36. Full Text

5. Abrishami A, Chan J, Chung F, Wong J. Preoperative pain sensitivity and its correlation with postoperative pain and analgesic consumption: a qualitative systematic review. Anesthesiology 2011;114:445-57. Full Text

6. Brandsborg B, Dueholm M, Kehlet H, Jensen TS, Nikolajsen L. Mechanosensitivity before and after hysterectomy: a prospective study on the prediction of acute and chronic postoperative pain. Br J Anaesth 2011;107:940-7. Full Text

7. Le Bars D, Dickenson AH, Besson JM. Diffuse noxious inhibitory controls (DNIC). I. Effects on dorsal horn convergent neurones in the rat. Pain 1979;6:283-304. Full Text

8. Le Bars D, Dickenson AH, Besson JM. Diffuse noxious inhibitory controls (DNIC). II. Lack of effect on non-convergent neurones, supraspinal involvement and theoretical implications. Pain 1979;6:30527. Full Text

9. Yarnitsky D, Arendt-Nielsen L, Bouhassira D, et al. Recommendations on terminology and practice of psychophysical DNIC testing. Eur J Pain 2010;14: 339. Full Text

10. De Broucker T, Cesaro P, Willer JC, Le Bars D. Diffuse noxious inhibitory controls in man. Involvement of the spinoreticular tract. Brain 1990;113(Pt 4):1223-34. Full Text

11. Willer JC, Le Bars D, De Broucker T. Diffuse noxious inhibitory controls in man: involvement of an opioidergic link. Eur J Pharmacol 1990;182:347-55. Full Text

12. Chitour D, Dickenson AH, Le Bars D. Pharmacological evidence for the involvement of serotonergic mechanisms in diffuse noxious inhibi- tory controls (DNIC). Brain Res 1982;236:329-37. Full Text

13. Pedersen LH, Nielsen AN, Blackburn-Munro G. Antinociception is selectively enhanced by parallel inhibition of multiple subtypes of monoamine transporters in rat models of persistent and neuropathic pain. Psychopharmacology (Berl) 2005;182:551-61. Full Text

14. Pertovaara A. Noradrenergic pain modulation. Prog Neurobiol 2006;80:53-83. Full Text

15. Iyengar S, Webster AA, Hemrick-Luecke SK, Xu JY, Simmons RM. Efficacy of duloxetine, a potent and balanced serotonin-norepinephrine reuptake inhibitor in persistent pain models in rats. J Pharmacol Exp Ther 2004;311:576-84. Full Text

16. Woolf CJ, Thompson SW. The induction and maintenance of central sensitization is dependent on N-methyl-D-aspartic acid receptor activation; implications for the treatment of post-injury pain hypersensitivity states. Pain 1991;44:293-9. Full Text

17. Kosek E, Hansson P. Modultory influence on somatosensory perception from vibration and heterotopic noxious conditioning stimulation (HNCS) in fibromyalgia and healthy subjects. Pain 1997;70:4151. Full Text

18. Lautenbacher S, Rollman GB. Possible deficiencies of pain modulation in fibromyalgia. Clin J Pain 1997;13: 189-96. Full Text

19. Price DD, Staud R. Neurobiology of fibromyalgia syndrome. J Rheumatol Suppl 2005;75:22-8. Review.

20. Staud R, Robinson ME, Price DD. Temporal summation of second pain and its maintenance are useful for characterizing widespread central sensitization of fibromyalgia patients. J Pain 2007;8:893-901. Full $\underline{\text { Text }}$

21. Staud R. Abnormal endogenous pain modulation is a shared characteristic of many chronic pain conditions. Expert Rev Neurother 2012;12:577-85 Full $\underline{\text { Text }}$

22. Julien N, Goffaux $P$, Arsenault $P$, Marchand S. Widespread pain in fibromyalgia is related to a deficit of endogenous pain inhibition. Pain 2005;114:295302. Full Text

23. Chang L. Brain responses to visceral and somatic stimuli in irritable bowel syndrome: a central nervous system disorder? Gastroenterol Clin North Am 2005;34:271-9. Full Text

24. Song GH, Venkatraman V, Ho KY, Chee NW, Yeoh KG, Wilder-Smith CH. Cortical effects of anticipation and endogenous modulation of visceral pain assessed by functional brain MRI in irritable bowel syndrome 
patients and healthy controls. Pain 2006;126:79-90. Full Text

25. King C, Wong F, Currie T, Mauderli A, Fillingim R, Riley J. Deficiency in endogenous modulation of prolonged heat pain in patients with irritable bowel syndrome and temporomandibular disorder. Pain 2009;143:172-8. Full Text

26. Sandrini G, Rossi P, Milanov I, Serrao M, Cecchini AP, Nappi G. Abnormal modulatory influence of diffuse noxious inhibitory controls in migraine and chronic tension-type headache patients. Cephalalgia 2006;26:782-9. Full Text

27. Buchgreitz L, Egsgaard LL, Jensen R, Arendt-Nielsen $\mathrm{L}$, Bendtsen L. Abnormal pain processing in chronic tension-type headache: a high-density EEG brain mapping study. Brain 2008;131:3232-8. Full Text

28. Pielsticker A, Haag G, Zaudig M, Lautenbacher S. Impairment of pain inhibition in chronic tension-type headache. Pain 2005;118:215-23. Full Text

29. Nahman-Averbuch H, Granovsky Y, Coghill RC, Yarnitsky D, Sprecher E, Weissman-Fogel I. Waning of "conditioned pain modulation": a novel expression of subtle pronociception in migraine. Headache 2013;53:1104-15. Full Text

30. Weissman-Fogel I, Sprecher E, Granovsky Y, Yarnitsky D. Repeated noxious stimulation of the skin enhances cutaneous pain perception of migraine patients in-between attacks: clinical evidence for continuous sub-threshold increase in membrane excitability of central trigeminovascular neurons. Pain 2003;104:693-700. Full Text

31. Maixner W, Fillingim R, Booker D, Sigurdsson A. Sensitivity of patients with painful temporomandibular disorders to experimentally evoked pain. Pain 1995;63:341-51. Full Text

32. Maixner W, Fillingim R, Sigurdsson A, Kincaid S, Silva S. Sensitivity of patients with painful temporomandibular disorders to experimentally evoked pain: evidence for altered temporal summation of pain. Pain 1998;76:71-81. Full Text

33. Sarlani E, Grace EG, Reynolds MA, Greenspan JD. Evidence for up-regulated central nociceptive processing in patients with masticatory myofascial pain. J Orofac Pain 2004;18:41-55.

34. Sarlani E, Garrett PH, Grace EG, Greenspan JD. Temporal summation of pain characterizes women but not men with temporomandibular disorders. J Orofac Pain2007;21:309-17.

35. Greenspan JD, Slade GD, Bair E, et al. Pain sensitivity risk factors for chronic TMD: descriptive data and empirically identified domains from the
OPPERA case control study. J Pain 2011;12(11 Suppl):T61-74. Full Text

36. Kosek E, Ordeberg G. Lack of pressure pain modulation by heterotopic noxious conditioning stimulation in patients with painful osteoarthritis before, but not following, surgical pain relief. Pain 2000;88:69-78. Full Text

37. Quante M, Hille S, Schofer MD, Lorenz J, Hauck M. Noxious counter irritation in patients with advanced osteoarthritis of the knee reduces MCC but not SII pain generators: a combined use of MEG and EEG. $J$ Pain Res 2008;1:1-8.

38. Arendt-Nielsen L, Nie $\mathrm{H}$, Laursen $\mathrm{MB}$, et al. Sensitization in patients with painful knee osteoarthritis. Pain 2010;149:573-81. Full Text

39. Graven-Nielsen T, Arendt-Nielsen L. Assessment of mechanisms in localized and widespread musculoskeletal pain. Nat Rev Rheumatol 2010;6:599-606. Full Text

40. Graven-Nielsen T, Wodehouse T, Langford RM, Arendt-Nielsen L, Kidd BL. Normalization of widespread hyperesthesia and facilitated spatial summation of deep-tissue pain in knee osteoarthritis patients after knee replacement. Arthritis Rheum 2012;64:2907-16. Full Text

41. Goodin BR, Glover TL, Sotolongo A, et al. The association of greater dispositional optimism with less endogenous pain facilitation is indirectly transmitted through lower levels of pain catastrophizing. $J$ Pain 2013;14:126-35. Full Text

42. Daenen L, Nijs J, Roussel N, Wouters K, Van Loo M, Cras P. Dysfunctional pain inhibition in patients with chronic whiplash-associated disorders: an experimental study. Clin Rheumatol 2013;32:23-31. Full Text

43. Lemming D, Graven-Nielsen T, Sörensen J, ArendtNielsen L, Gerdle B. Widespread pain hypersensitivity and facilitated temporal summation of deep tissue pain in whiplash associated disorder: an explorative study of women. J Rehabil Med 2012;44: 648-57. Full Text

44. Van Oosterwijck J, Nijs J, Meeus M, Paul L. Evidence for central sensitization in chronic whiplash: a systematic literature review. Eur J Pain 2013;17:299312. Full Text

45. Yarnitsky D, Crispel Y, Eisenberg E, et al. Prediction of chronic post-operative pain: pre-operative DNIC testing identifies patients at risk. Pain 2008;138:228. Full Text

46. Landau R, Kraft JC, Flint LY, et al. An experimental paradigm for the prediction of post-operative pain (PPOP). J Vis Exp 2010;(35):1671. 
47. Wilder-Smith OH, Schreyer T, Scheffer GJ, ArendtNielsen L. Patients with chronic pain after abdominal surgery show less preoperative endogenous pain inhibition and more postoperative hyperalgesia: a pilot study. J Pain Palliat Care Pharmacother 2010; 24:119-28. Full Text

48. De Felice M, Sanoja R, Wang R, et al. Engagement of descending inhibition from the rostral ventromedial medulla protects against chronic neuropathic pain. Pain 2011;152:2701-9. Full Text

49. Yarnitsky D, Granot M, Nahman-Averbuch $H$, Khamaisi M, Granovsky Y. Conditioned pain modulation predicts duloxetine efficacy in painful diabetic neuropathy. Pain 2012;153:1193-8. Full Text

50. Lavand'homme P, Roelants F. Effect of a low dose of ketamine on postoperative pain after elective Cesarean delivery according to the presence of a preoperative temporal summation. 2009; SOAP abstract A-258.

51. Lindstedt F, Berrebi J, Greayer E, et al. Conditioned pain modulation is associated with common poly- morphisms in the serotonin transporter gene. PLoS One 2011;6:e18252. Full Text

52. Treister R, Pud D, Ebstein RP, et al. Association between polymorphisms in serotonin and dopaminerelated genes and endogenous pain modulation. $\mathrm{J}$ Pain 2011;12:875-83. Full Text

53. Lev R, Granovsky Y, Yarnitsky D. Orbitofrontal disinhibition of pain in migraine with aura: an interictal EEG-mapping study. Cephalalgia 2010; 30:910-18.

54. Sprenger C, Bingel U, Büchel C. Treating pain with pain: supraspinal mechanisms of endogenous analgesia elicited by heterotopic noxious conditioning stimulation. Pain 2011;152:428-39. Full Text

55. Loggia ML, Jensen $\mathrm{K}$, Gollub RL, Wasan AD, Edwards RR, Kong J. The catechol-O-methyltransferase (COMT) val158met polymorphism affects brain responses to repeated painful stimuli. PLoS One 2011;6:e27764. Full Text 